\title{
ABSTRACT MULTISCALE-HYBRID-MIXED METHODS
}

\author{
ALEXANDRE L. MADUREIRA
}

Dedicated to Leo Franca, in memoriam.

\begin{abstract}
In an abstract setting, we investigate the basic ideas behind the Multiscale Hybrid Mixed (MHM) method, a Domain Decomposition scheme designed to solve multiscale partial differential equations (PDEs) in parallel. As originally proposed, the MHM method starting point is a primal hybrid formulation, which is then manipulated to result in an efficient method that is based on local independent PDEs and a global problem that is posed on the skeleton of the finite element mesh. Recasting the MHM method in a more general framework, we investigate some conditions that yield a well-posed method. We apply the general ideas to different formulations, and, in particular, come up with an interesting and fruitful connection between the Multiscale Finite Element Method and a dual hybrid method. Finally, we propose a method that combines the main ideas of the Discontinuous Enrichment Method and the MHM method.
\end{abstract}

\section{THE ORIGINAL PROBLEM}

Multiscale problems are, by definition, difficult to solve computationally, and numerical methods with domain decomposition flavor are appealing, since they woo parallel implementations. Some important ideas have been around for a while, for instance in the seminal paper [20]. More recent attempts to solve multiscale PDEs using domain decomposition or hybrid formulations include $[2,3,15,19]$. Several theoretical aspects and applications involving hybrid methods appear, for instance, in $[4,11-13,26]$, and references therein.

An interesting proposal by Harder, Paredes and Valentin [23] came out also recently. Aiming to solve heterogeneous Darcy equations using a primal hybrid method, the authors came up with an efficient formulation that is well-posed and easy to implement in parallel. They christened the scheme Multiscale Hybrid Mixed, or MHM for short.

Date: September 25, 2014.

Key words and phrases. MHM, mixed method, hybrid method, domain decomposition, finite element, multiscale.

We thank Frédéric Valentin for several fruitful discussions and comments that enriched the present work. We also gratefully acknowledge the partial support of CNPq, grant numbers 308360/2010-9 and 560108/2010-9. 
The MHM method was latter extended to other operators [1,22,24,28], but it was always developed in a case by case basis. The aim of the present work is to state the method in a more general form, and show that, under certain conditions, the scheme is well-posed. We also explore applications of the method from different starting points, and in particular an interesting connection between a dual hybrid formulation and the Multiscale Finite Element methods pops up. Finally, we explore ideas of the Discontinuous Enrichment Method, recasting them in the MHM method formalism.

The remainder of this section contains the definition of a mixed formulation in Hilbert spaces, and some working hypothesis. Next, Section 2 deals with the development and analysis of the MHM method in a continuous setting, while Section 3 does the same in a discrete setting. Finally, some examples are presented in Section 4, and Section 5 unfolds a method that combines the MHM and the Discontinuous Enrichment Methods.

We consider mixed methods for PDEs in Hilbert Spaces. For theoretical background see [5, 6, $16,29,30]$. Let $X, M$ be Hilbert spaces, and $a \in \mathcal{L}(X \times X, \mathbb{R}), b \in \mathcal{L}(X \times M, \mathbb{R}), l \in X$ and $\sigma \in M$. The symbol $\mathcal{L}\left(H_{1}, H_{2}\right)$ denotes the space of continuous linear functions between Hilbert spaces $H_{1}$ and $H_{2}$.

Consider the problem of finding $(u, \eta) \in X \times M$ such that

$$
\begin{array}{ll}
a(u, v)+b(v, \eta)=l(v) & \text { for all } v \in X, \\
b(u, \mu) & =\sigma(\mu)
\end{array}
$$

We interpret $l(\cdot)$ as an action of $l \in X$ through the $X$-inner-product. Similarly for $\sigma(\cdot)$, etc. We could also consider the same problem in the form

$$
\begin{aligned}
& A u+B^{T} \eta=l \quad \text { in } X \\
& B u=\sigma \text { in } M \text {, }
\end{aligned}
$$

where $A \in \mathcal{L}(X, X)$ and $B \in \mathcal{L}(X, M)$ are such that

$$
\langle A w, v\rangle_{X}=a(w, v), \quad\langle B v, \mu\rangle_{M}=b(v, \mu)
$$

for all $v, w \in X$ and $\mu \in M$. Here, $\langle\cdot, \cdot\rangle_{X}$ and $\langle\cdot, \cdot\rangle_{M}$ denote the $X$ - and $M$-inner-products.

To proceed with the description of the method we consider some assumptions, which in particular guarantee that (1) is well-posed. It is convenient to introduce some notation at this point. Let 
$X_{\mathrm{rm}}$ be the space of "rigid motions", elements that belong to the kernel of $A$ :

$$
X_{\mathrm{rm}}=\operatorname{Kern} A=\{w \in X: a(w, v)=0 \text { for all } v \in X\},
$$

and let

$$
X=X_{\mathrm{rm}} \oplus \tilde{X}
$$

where $\tilde{X}=X_{\text {rm }}^{\perp}$ with respect to the $X$-inner-product.

\section{Hypotheses 1.}

(a) a $(\cdot, \cdot)$ is symmetric.

(b) Usual well-posedness assumptions for (1):

$$
a(v, v) \gtrsim\|v\|_{X}^{2} \text { for } v \in \operatorname{Kern}(B), \quad \inf _{\mu \in M} \sup _{v \in X} \frac{b(v, \mu)}{\|v\|_{X}\|\mu\|_{M}} \gtrsim 1 .
$$

(c) Korn-like inequality: $a(\cdot, \cdot)$ is coercive in $\tilde{X}$

$$
a(\tilde{v}, \tilde{v}) \gtrsim\|\tilde{v}\|_{X}^{2} \quad \text { for all } \tilde{v} \in \tilde{X} .
$$

The symbols $\gtrsim$ and $\lesssim$ indicate that the corresponding inequalities hold up to constants.

\section{MHM: CONTINUOUS SETTING}

We divide this section in two parts. The first part contains the formal derivation of the method. The second part tells us that not only all the formal steps are valid, but also that the final problem is well-posed.

2.1. The formalism. From (3), given $u \in X$ there are unique $u_{\mathrm{rm}} \in X_{\mathrm{rm}}$ and $\tilde{u} \in \tilde{X}$ such that $u=u_{\mathrm{rm}}+\tilde{u}$. We gather from (1) and the definition of $X_{\mathrm{rm}}$ that

$$
\begin{aligned}
& a(\tilde{u}, \tilde{v}) \quad+b(\tilde{v}, \eta) \quad=l(\tilde{v}) \quad \text { for all } \tilde{v} \in \tilde{X}, \\
& b\left(v_{\mathrm{rm}}, \eta\right)=l\left(v_{\mathrm{rm}}\right) \quad \text { for all } v_{\mathrm{rm}} \in X_{\mathrm{rm}}, \\
& b(\tilde{u}, \mu)+b\left(u_{\mathrm{rm}}, \mu\right) \quad=\sigma(\mu) \quad \text { for all } \mu \in M .
\end{aligned}
$$

Let $T: M \rightarrow \tilde{X}$ and $\hat{T}: X \rightarrow \tilde{X}$ such that

$$
a(T \eta, \tilde{v})=b(\tilde{v}, \eta), \quad a(\hat{T} l, \tilde{v})=l(\tilde{v}), \quad \text { for all } \tilde{v} \in \tilde{X} .
$$


Thus

$$
\tilde{u}=-T \eta+\hat{T} l
$$

and we gather then that $u_{\mathrm{rm}} \in X_{\mathrm{rm}}$ and $\eta \in M$ solve

$$
\begin{array}{rlrl}
b(T \eta, \mu)-b\left(u_{\mathrm{rm}}, \mu\right) & =-\sigma(\mu)+b(\hat{T} l, \mu) & & \text { for all } \mu \in M, \\
b\left(v_{\mathrm{rm}}, \eta\right) & =l\left(v_{\mathrm{rm}}\right) & \text { for all } v_{\mathrm{rm}} \in X_{\mathrm{rm}} .
\end{array}
$$

In terms of operators, the above splitting leads to

$$
\begin{aligned}
\tilde{A} \tilde{u}+\tilde{B}^{T} \eta & =\tilde{l}, \\
B_{\mathrm{rm}}^{T} \eta & =l_{\mathrm{rm}}, \\
\tilde{B} \tilde{u}+B_{\mathrm{rm}} u_{\mathrm{rm}} & =\sigma,
\end{aligned}
$$

where $\tilde{l} \in \tilde{X}, l_{\mathrm{rm}} \in X_{\mathrm{rm}}, \tilde{A} \in \mathcal{L}(\tilde{X}, \tilde{X}), \tilde{B} \in \mathcal{L}(\tilde{X}, M)$ and $B_{\mathrm{rm}} \in \mathcal{L}\left(X_{\mathrm{rm}}, M\right)$ are such that

$$
\begin{gathered}
\tilde{l}(w)=l(w) \quad \text { for all } w \in \tilde{X}, \quad l_{\mathrm{rm}}(w)=l(w) \quad \text { for all } w \in X_{\mathrm{rm}}, \\
\langle\tilde{A} w, v\rangle=a(w, v) \quad \text { for all } w, v \in \tilde{X} \\
\langle\tilde{B} v, \mu\rangle=b(v, \mu) \quad \text { for all } v \in \tilde{X}, \mu \in M \\
\left\langle B_{\mathrm{rm}} w, \mu\right\rangle=b(w, \mu) \quad \text { for all } w \in X_{\mathrm{rm}}, \mu \in M
\end{gathered}
$$

With such notation, $T=\tilde{A}^{-1} \tilde{B}^{T}$ and $\hat{T}=\tilde{A}^{-1}$. Using (5),

$$
\begin{aligned}
\tilde{B} \tilde{A}^{-1} \tilde{B}^{T} \eta-B_{\mathrm{rm}} u_{\mathrm{rm}} & =-\sigma+\tilde{B} \tilde{A}^{-1} \tilde{l}, \\
B_{\mathrm{rm}}^{T} \eta \quad & =l_{\mathrm{rm}} .
\end{aligned}
$$

Remark 2. In the case where $A$ is invertible, then $\tilde{B} \tilde{A}^{-1} \tilde{B}^{T}=B A^{-1} B^{T}$ is often called Uzawa operator and Schur complement.

The whole purpose of the MHM method is that there are instances where efficient computations of $T, \hat{T}$ are feasible, possibly in parallel, and the resulting system (6) (or (8)) is of reduced size. Of course, whenever $A$ is injective, then $X_{\mathrm{rm}}=\{0\}$ and the final system is no longer of mixed type, but elliptic, simplifying matters even further. 
2.2. Mathematical properties. The well-posedness of (4) follows from the assumption (c) in Hypotheses 1. This also guarantees that $T$ and $\hat{T}$ are well-defined, and that these operators are coercive and bounded.

Now, the existence of solution for (6) (or (8)), follows from the well-posedness of (1). Indeed, given the solution pair $(u, \eta) \in X \times M$ we decompose $u=\tilde{u}+u_{\text {rm }}$ and gather that (5) holds. Thus, the first equation of (6) is satisfied. The second equation in (6) follows immediately from the first equation in (1).

Reversing the steps, if $\left(u_{\mathrm{rm}}, \eta\right) \in X_{\mathrm{rm}} \times M$ solves (6) with zero right hand side, then a simple substitution yields that $u=-T \eta+u_{\mathrm{rm}}$ and $\eta$ satisfy (1) also with zero right hand side. So, uniqueness of solutions for (6) follows.

The well-posedness of (6) or its equivalent form (8) can also be derived directly. Note first that $b(T \cdot, \cdot)$ is actually symmetric positive definite, since from (4),

$$
b(T \eta, \mu)=a(T \mu, T \eta)
$$

and from Hypothesis 1.c,

$$
b(T \mu, \mu)=a(T \mu, T \mu) \gtrsim\|T \mu\|_{X}^{2} \gtrsim\|\mu\|_{M}^{2} .
$$

Showing the inf-sup condition

$$
\sup _{\mu \in M} \frac{b\left(v_{\mathrm{rm}}, \mu\right)}{\|\mu\|_{M}} \gtrsim\left\|v_{\mathrm{rm}}\right\|_{X},
$$

is equivalent [16, page 470] to show that $B_{\mathrm{rm}}$ is one to one and that $\operatorname{Im}\left(B_{\mathrm{rm}}\right)$ is closed in $M$. That is the content of the following lemma.

Lemma 3. Let $B_{\mathrm{rm}}$ be defined as in (7), and assume that the Hypotheses 1 hold. Then $B_{\mathrm{rm}}$ is injective and $\mathrm{Im} B_{\mathrm{rm}}$ is closed in $M$.

Proof. Let $v_{\mathrm{rm}} \in \operatorname{Kern} B_{\mathrm{rm}} \subset X_{\mathrm{rm}}$. Then $v_{\mathrm{rm}} \in \operatorname{Kern} B$ and the first inequality in Hypothesis 1.b yields $\left\|v_{\mathrm{rm}}\right\|_{X}^{2} \lesssim a\left(v_{\mathrm{rm}}, v_{\mathrm{rm}}\right)=0$ since $v_{\mathrm{rm}} \in X_{\mathrm{rm}}$. Thus $v_{\mathrm{rm}}=0$, and $B_{\mathrm{rm}}$ is one to one.

To show that $\operatorname{Im} B_{\mathrm{rm}}$ is closed in $M$, let $\mu_{n} \rightarrow \mu$ in $M$ such that $\mu_{n} \in \operatorname{Im} B_{\mathrm{rm}} \subset \operatorname{Im} B$. From the second inequality of Hypothesis $1 . \mathrm{b}$ it follows that $B$ is surjective, and thus there exist $v_{n} \in X$ such that $B v_{n}=\mu_{n}$ and $\left\|v_{n}\right\|_{X} \lesssim\left\|\mu_{n}\right\|_{M}$. Let $v_{\mathrm{rm}}^{n} \in X_{\mathrm{rm}}$ such that $v_{\mathrm{rm}}^{n}-v_{n} \in \operatorname{Kern} B$. Then, from (b),

$$
\left\|v_{\mathrm{rm}}^{n}\right\|_{X} \lesssim\left\|v_{\mathrm{rm}}^{n}-v_{n}\right\|_{X}+\left\|v_{n}\right\|_{X} \lesssim a\left(v_{n}, v_{n}\right)^{1 / 2}+\left\|v_{n}\right\|_{X} \lesssim\left\|v_{n}\right\|_{X} \lesssim\left\|\mu_{n}\right\|_{M}
$$


Since $\mu_{n}$ converges, $\left\|v_{\mathrm{rm}}^{n}\right\|_{X}$ must be bounded. Let then $v_{\mathrm{rm}} \in X_{\mathrm{rm}}$ be the weak limit of a converging subsequence of $\left(v_{\mathrm{rm}}^{n}\right)$, which we still denote by $\left(v_{\mathrm{rm}}^{n}\right)$. Then

$$
\left\langle B_{\mathrm{rm}} v_{\mathrm{rm}}, \eta\right\rangle=\left\langle v_{\mathrm{rm}}, B_{\mathrm{rm}}^{T} \eta\right\rangle=\lim _{n \rightarrow \infty}\left\langle B_{\mathrm{rm}} v_{\mathrm{rm}}^{n}, \eta\right\rangle=\lim _{n \rightarrow \infty}\left\langle\mu_{n}, \eta\right\rangle=\langle\mu, \eta\rangle
$$

for all $\eta \in M$. Thus $\mu=B_{\mathrm{rm}} v_{\mathrm{rm}} \in \operatorname{Im} B_{\mathrm{rm}}$, and $\operatorname{Im} B_{\mathrm{rm}}$ is closed.

\section{MHM: DISCRETE SETTING}

There are basically two ways to discretize (1). One is to simply discretize (6), and that was done in the original proposal [23] and latter in [22,28]. However it is also possible to first discretize (1) and only then perform the decomposition (3). Under a mild restriction, it turns out that these approaches are actually equivalent.

3.1. Discretize first, split spaces latter. Starting with (1), we define $X_{h} \subset X$ and $M_{h} \subset M$, and search for $\left(u^{h}, \eta^{h}\right) \in X_{h} \times M_{h}$ such that

$$
\begin{aligned}
a\left(u^{h}, v^{h}\right)+b\left(v^{h}, \eta\right) & =l\left(v^{h}\right) \quad \text { for all } v^{h} \in X_{h} \\
b\left(u^{h}, \mu^{h}\right) & =\sigma\left(\mu^{h}\right) \quad \text { for all } \mu \in M_{h} .
\end{aligned}
$$

We assume that the discrete counterpart of Hypothesis 1.b holds, and then (9) is well-posed. Assuming also that $X_{\mathrm{rm}} \subset X_{h}$, we can mimic (3) and decompose $X_{h}=X_{\mathrm{rm}} \oplus \tilde{X}_{h}$, again with respect to the $X$-inner-product. Let $u^{h}=u_{\mathrm{rm}}^{h}+\tilde{u}^{h} \in X_{\mathrm{rm}} \oplus \tilde{X}_{h}$, and proceeding as in Section 2 we obtain

$$
\begin{array}{rlrl}
b\left(T_{h} \eta^{h}, \mu^{h}\right)-b\left(u_{\mathrm{rm}}^{h}, \mu^{h}\right) & =-\sigma\left(\mu^{h}\right)+b\left(\hat{T}_{h} l^{h}, \mu^{h}\right) & & \text { for all } \mu^{h} \in M_{h}, \\
b\left(v_{\mathrm{rm}}, \eta^{h}\right) & =l\left(v_{\mathrm{rm}}\right) & \text { for all } v_{\mathrm{rm}} \in X_{\mathrm{rm}},
\end{array}
$$

where $l^{h}$ is the projection of $l$ in $\tilde{X}_{h}$. As in (4), $T_{h}: M_{h} \rightarrow \tilde{X}_{h}$ and $\hat{T}_{h}: \tilde{X}_{h} \rightarrow \tilde{X}_{h}$ solve

$$
a\left(T_{h} \eta, \tilde{v}\right)=b(\tilde{v}, \eta), \quad a\left(\hat{T}_{h} l^{h}, \tilde{v}\right)=l^{h}(\tilde{v}), \quad \text { for all } \tilde{v} \in \tilde{X}_{h} .
$$

Similarly to the continuous case, $T_{h}=\tilde{A}_{h}^{-1} \tilde{B}_{h}^{T}$ and $\hat{T}_{h}=\tilde{A}_{h}^{-1}$, where $\tilde{A}_{h}$ and $\tilde{B}_{h}$ are the discrete counterparts of $\tilde{A}$ and $\tilde{B}$. The existence of $\tilde{A}_{h}^{-1}$ follows from Hypothesis 1.c, and the matrix formulation of (10) follows (8). Observe that (10) is then nothing more than (9), written differently. Thus, (10) is also well-posed and we have the error estimate

$$
\left\|\eta-\eta^{h}\right\|_{M}+\left\|u-u^{h}\right\|_{X} \lesssim \inf _{v^{h} \in X_{h}}\left\|u-v^{h}\right\|_{X}+\inf _{\mu^{h} \in M_{h}}\left\|\eta-\mu^{h}\right\|_{M}
$$


Under the extra hypotheses that $T_{h}=T$ and $\hat{T}=\hat{T}_{h}$ then

$$
\inf _{v^{h} \in X_{h}}\left\|u-v^{h}\right\|_{X} \leq \inf _{\tilde{v}^{h} \in \tilde{X}_{h}}\left\|\tilde{u}-\tilde{v}^{h}\right\|_{X} \leq \inf _{\mu^{h} \in M_{h}}\left\|T \eta-T \mu^{h}\right\|_{X} \lesssim \inf _{\mu^{h} \in M_{h}}\left\|\eta-\mu^{h}\right\|_{X} .
$$

Thus, (12) becomes

$$
\left\|\eta-\eta^{h}\right\|_{M}+\left\|u-u^{h}\right\|_{X} \lesssim \inf _{\mu^{h} \in M_{h}}\left\|\eta-\mu^{h}\right\|_{M}
$$

Remark 4. The estimate (13) was obtained in $[1,22]$ for the applications considered in those papers, and states that the numerical error for the method is controlled by the discretization at the mesh skeleton. The influence of local discretizations was also considered in [22]. Of course, several factor can contribute to deteriorate the estimate, since the constants can depend on physical parameters, and even on the mesh, if the discrete inf-sup conditions depend on $h$. These situations have to be examined and healed in a case-by-case basis.

3.2. Split spaces first, discretize latter. We now take a different standing point, first splitting the spaces to obtain (6). The discretion step comes out by choosing $M_{h} \subset M$, but we assume that $X_{\mathrm{rm}}$ is so simple (finite dimensional) that we have it analytically. Adding a zest to the model, we allow the possibility that $T$ and $\hat{T}$ are approximated by a different scheme other than choosing $X_{h} \subset X$ to discretize (4). Thus, we seek $u_{\mathrm{rm}}^{h} \in X_{\mathrm{rm}}$ and $\eta^{h} \in M_{h}$ such that

$$
\begin{array}{rlrl}
b\left(\mathfrak{T}_{h} \eta^{h}, \mu^{h}\right)-b\left(u_{\mathrm{rm}}^{h}, \mu^{h}\right) & =-\sigma\left(\mu^{h}\right)+b\left(\hat{\mathfrak{T}}_{h} l^{h}, \mu^{h}\right) & & \text { for all } \mu^{h} \in M_{h}, \\
b\left(v_{\mathrm{rm}}, \eta^{h}\right) & =l\left(v_{\mathrm{rm}}\right) & \text { for all } v_{\mathrm{rm}} \in X_{\mathrm{rm}},
\end{array}
$$

where $\mathfrak{T}_{h}, \hat{\mathfrak{T}}_{h}$ indicate stable approximations for $T, \hat{T}$, defined in (4). Thus, $b\left(\mathfrak{T}_{h}, \cdot\right)$ is coercive since

$$
b\left(\mathfrak{T}_{h} \mu^{h}, \mu^{h}\right)=a\left(\mathfrak{T}_{h} \mu^{h}, \mathfrak{T}_{h} \mu^{h}\right) \gtrsim\left\|\mathfrak{T}_{h} \mu^{h}\right\|_{X} \gtrsim\left\|\mu^{h}\right\|_{M} .
$$

The well-posedness of (14) follows under the inf-sup assumption

$$
\inf _{v_{\mathrm{rm}} \in X_{\mathrm{rm}}} \sup _{\mu^{h} \in M_{h}} \frac{b\left(v_{\mathrm{rm}}, \mu^{h}\right)}{\left\|v_{\mathrm{rm}}\right\|_{X}\left\|\mu^{h}\right\|_{M}} \gtrsim 1 .
$$

With the aid of Strang's Lemma, it is possible to bound the error as follows $[6,16]$ :

$$
\left\|\eta-\eta^{h}\right\|_{M}+\left\|u_{\mathrm{rm}}-u_{\mathrm{rm}}^{h}\right\|_{X} \lesssim \inf _{\mu^{h} \in M_{h}}\left\|\eta-\mu^{h}\right\|_{M}+\left\|T-\mathfrak{T}_{h}\right\|_{\mathcal{L}(M, \tilde{X})}+\left\|\hat{T}-\hat{\mathfrak{T}}_{h}\right\|_{\mathcal{L}(X, \tilde{X})}
$$

Defining $\tilde{u}^{h}=-\mathfrak{T}_{h} \eta^{h}+\hat{\mathfrak{T}}_{h} l$ and $u^{h}=u_{\mathrm{rm}}^{h}+\tilde{u}^{h}$, we gather that $\left\|\tilde{u}-\tilde{u}^{h}\right\|_{X} \leq\left\|T \eta-\mathfrak{T}_{h} \eta^{h}\right\|_{X}+\left\|\hat{T} l-\hat{\mathfrak{T}}_{h} l\right\|_{X} \leq\left\|\eta-\eta^{h}\right\|_{X}+\left\|T-\mathfrak{T}_{h}\right\|_{\mathcal{L}(M, \tilde{X})}+\left\|\hat{T}-\hat{\mathfrak{T}}_{h}\right\|_{\mathcal{L}(X, \tilde{X})}$, 
Thus

$$
\left\|\eta-\eta^{h}\right\|_{M}+\left\|u-u^{h}\right\|_{X} \lesssim \inf _{\mu^{h} \in M_{h}}\left\|\eta-\mu^{h}\right\|_{M}+\left\|T-\mathfrak{T}_{h}\right\|_{\mathcal{L}(M, \tilde{X})}+\left\|\hat{T}-\hat{\mathfrak{T}}_{h}\right\|_{\mathcal{L}(X, \tilde{X})}
$$

Remark 5. In terms of implementation, it is necessary to compute the action of $T$ on a basis of $M_{h}$. Consider then $\left\{\psi_{i}\right\}_{i=1}^{\operatorname{dim} M_{h}}$ a basis for $M_{h}$, define $\lambda_{i}=-T \psi_{i}$, and assume that $\eta=\sum_{i=1}^{\operatorname{dim} M_{h}} \eta_{i} \psi_{i}$. This allows the computations of the matrices involved in (10), and the post-processing $u=u_{\mathrm{rm}}+$ $\sum_{i=1}^{\operatorname{dim} M_{h}} \eta_{i} \lambda_{i}+\hat{T} l$.

Remark 6. The approximation of $T$ is a matter of choice, and, in particular, another round of a MHM method at the local level should not be ruled out, originating a sort of numerical zoom (Frédéric Valentin, personal communication, 2013). This however, becomes clear only in the split first, discretize latter scheme. If choices of discretizations for $X$ are done beforehand (see previous subsection), then $T_{h}$ is uniquely defined, and $\mathfrak{T}_{h}=T_{h}=\tilde{A}_{h}^{-1} \tilde{B}_{h}^{T}$ and $\hat{\mathfrak{T}}_{h}=\hat{T}_{h}=\tilde{A}_{h}^{-1}$.

\section{Some EXAMPLES}

In this section we explore how the general ideas previously developed can be applied to wellknown formulations of a simple elliptic problem. Briefly, what we obtain is that starting from the dual and primal mixed formulation, we either cannot apply the formalism (dual mixed formulation) or, depending on the local discretization, obtain the Galerkin method (primal mixed formulation). The hybrid formulations yield interesting cases, delivering either the method of [23] (primal hybrid formulation), or the Multiscale Finite Element Method (dual hybrid formulation).

For a polygonal domain $\Omega \subset \mathbb{R}^{2}$, and $\mathcal{C}: \Omega \rightarrow \mathbb{R}_{\text {sym }}^{2 \times 2}$, consider the Poisson problem of finding $u: \Omega \rightarrow \mathbb{R}$ and $\sigma: \Omega \rightarrow \mathbb{R}^{2}$ weak solution of

$$
\boldsymbol{\sigma}=\mathcal{C} \boldsymbol{\nabla} u, \quad-\operatorname{div} \boldsymbol{\sigma}=f \quad \text { in } \Omega, \quad u=0 \quad \text { on } \partial \Omega .
$$

In what follows, we apply the MHM method formalism to different mixed formulations, starting with the traditional primal and mixed formulations, and proceeding to the hybrid forms. 
4.1. Dual mixed formulation. Characterizing the above problem from the second HellingerReissner principle yields that $\boldsymbol{\sigma} \in \boldsymbol{H}(\operatorname{div} ; \Omega)$ and $u \in L^{2}(\Omega)$ solve

$$
\begin{aligned}
\int_{\Omega} \mathcal{C}^{-1} \boldsymbol{\sigma} \cdot \boldsymbol{\tau} d \boldsymbol{x}+\int_{\Omega} u \operatorname{div} \boldsymbol{\tau} d \boldsymbol{x} & =0 \quad \text { for all } \boldsymbol{\tau} \in \boldsymbol{H}(\operatorname{div} ; \Omega) \\
\int_{\Omega} \operatorname{div} \boldsymbol{\sigma} v d \boldsymbol{x} & =-\int_{\Omega} f v d \boldsymbol{x} \quad \text { for all } v \in L^{2}(\Omega) .
\end{aligned}
$$

Using the notation previously introduced, we gather that $a(\boldsymbol{\sigma}, \boldsymbol{\tau})=\int_{\Omega} \mathcal{C}^{-1} \boldsymbol{\sigma} \cdot \boldsymbol{\tau} d \boldsymbol{x}$ is not coercive over $\tilde{X}=\boldsymbol{H}(\operatorname{div} ; \Omega)$, violating the Hypothesis 1.c. That, of course, does not mean that the dual mixed formulation is not well-posed. It means only that we cannot apply the MHM method formalism as developed in Section 2.

4.2. Primal mixed formulation. Another characterization searches for $\boldsymbol{\sigma} \in \boldsymbol{L}^{2}(\Omega)$ and $u \in$ $\stackrel{\circ}{H}^{1}(\Omega)$ solve

$$
\begin{aligned}
\int_{\Omega} \mathcal{C}^{-1} \boldsymbol{\sigma} \cdot \boldsymbol{\tau} d \boldsymbol{x}-\int_{\Omega} \boldsymbol{\nabla} u \cdot \boldsymbol{\tau} d \boldsymbol{x} & =0 \quad \text { for all } \boldsymbol{\tau} \in \boldsymbol{L}^{2}(\Omega) \\
\int_{\Omega} \boldsymbol{\sigma} \cdot \boldsymbol{\nabla} v d \boldsymbol{x} & =\int_{\Omega} f v d \boldsymbol{x} \quad \text { for all } v \in \stackrel{\circ}{H}^{1}(\Omega) .
\end{aligned}
$$

Using the notation previously introduced,

$$
\begin{gathered}
a(\boldsymbol{\sigma}, \boldsymbol{\tau})=\int_{\Omega} \mathcal{C}^{-1} \boldsymbol{\sigma} \cdot \boldsymbol{\tau} d \boldsymbol{x}, \quad b(\boldsymbol{\tau}, v)=-\int_{\Omega} \boldsymbol{\nabla} v \cdot \boldsymbol{\tau} d \boldsymbol{x}, \\
X=\tilde{X}=\boldsymbol{L}^{2}(\Omega), \quad X_{\mathrm{rm}}=\{0\}, \quad M=\stackrel{\circ}{H}^{1}(\Omega) .
\end{gathered}
$$

In the present case, $T: \stackrel{\circ}{H}^{1}(\Omega) \rightarrow \boldsymbol{L}^{2}(\Omega)$ is such that, for $v \in \stackrel{\circ}{H}^{1}(\Omega)$,

$$
\int_{\Omega} \mathcal{C}^{-1}(T v) \cdot \boldsymbol{\tau} d \boldsymbol{x}=-\int_{\Omega} \boldsymbol{\nabla} v \cdot \boldsymbol{\tau} d \boldsymbol{x}
$$

for all $\boldsymbol{\tau} \in \boldsymbol{L}^{2}(\Omega)$. Thus $T v=-\mathcal{C} \nabla v$, and the MHM method seeks $u \in \stackrel{\circ}{H}^{1}(\Omega)$ such that

$$
\int_{\Omega} T u \cdot \nabla v d \boldsymbol{x}=-\int_{\Omega} f v d \boldsymbol{x} \quad \text { for all } v \in \stackrel{\circ}{H}^{1}(\Omega) .
$$

So, the application of the MHM method formalism simply yield the primal Galerkin method at the continuous level. At the discrete level, the method will depend on the discretization of $T$. 
4.3. Primal hybrid formulation. We shall not discuss this case in detail since it was extensively described already [23]. We mention however that after partitioning $\Omega$ into elements, the resulting spaces are: $X_{\mathrm{rm}}$ composed of piecewise constants, $\tilde{X}$ the space of functions that are elementwise in $H^{1}$, and $M$ is the space $H^{-1 / 2}$ on the mesh skeleton. The operator $T$ returns solutions of local problems with Neumann boundary conditions. This is not only an embarrassingly parallel [32] scheme, but it also avoids the onset of strong spurious internal boundary layers in the presence of heterogeneous coefficients $[25,31]$. The final problem (6) is posed on the mesh skeleton, and is cheap to solve.

A peculiarity of the MHM method is that decomposition of $X$ made the local problems wellposed, making the primal hybrid formulation, with all its natural advantages, as easy to implement as its dual counterpart, avoiding difficulties as discussed in [27]. This sort of approach is not limited to problems in the form (6). In [21] for instance, a similar situation arises, depending on geometric conditions of the domain partition.

4.4. Dual hybrid formulation. We now formulate the problem using the dual hybrid formulation $[2,3,5,10,29]$.

4.4.1. Continuous formulation. Let $\mathcal{P}$ be a partition of $\Omega$ into elements, and let $\mathcal{E}$ be the mesh skeleton associated with $\mathcal{P}$. Consider the spaces associated to such partition,

$$
\mathbf{W}^{f}=\left\{\boldsymbol{\tau} \in \boldsymbol{L}^{2}(\Omega):-\operatorname{div} \boldsymbol{\tau}=f \text { in } K \in \mathcal{P}\right\}, \quad H^{1 / 2}(\mathcal{E})=\left\{\left.v\right|_{\mathcal{E}}: v \in \stackrel{\circ}{H}^{1}(\Omega)\right\}
$$

Fixing $\boldsymbol{\sigma}^{f} \in \mathbf{W}^{f}$ arbitrarily, we seek then $\boldsymbol{\sigma} \in \mathbf{W}^{0}$ and $u \in H^{1 / 2}(\mathcal{E})$ such that

$$
\begin{aligned}
\int_{\Omega} \mathcal{C}^{-1} \boldsymbol{\sigma} \cdot \boldsymbol{\tau} d \boldsymbol{x}-\sum_{K \in \mathcal{P}} \int_{\partial K} u \boldsymbol{\tau} \cdot \mathbf{n} d \boldsymbol{x} & =-\int_{\Omega} \mathcal{C}^{-1} \boldsymbol{\sigma}^{f} \cdot \boldsymbol{\tau} d \boldsymbol{x} & \text { for all } \boldsymbol{\tau} \in \mathbf{W}^{0}, \\
-\sum_{K \in \mathcal{P}} \int_{\partial K} v \boldsymbol{\sigma} \cdot \mathbf{n} d \boldsymbol{x} & =\sum_{K \in \mathcal{P}} \int_{\partial K} v \boldsymbol{\sigma}^{f} \cdot \mathbf{n} d \boldsymbol{x} & \text { for all } v \in H^{1 / 2}(\mathcal{E}) .
\end{aligned}
$$

It is not hard to check that the solutions of (16) satisfy the above system, but a caveat is necessary: in (16), $u$ belongs to $\stackrel{\circ}{H}^{1}(\Omega)$, while here we are considering its trace only. In terms of the MHM 
method,

$$
\begin{gathered}
a(\boldsymbol{\sigma}, \boldsymbol{\tau})=\int_{\Omega} \mathcal{C}^{-1} \boldsymbol{\sigma} \cdot \boldsymbol{\tau} d \boldsymbol{x}, \quad b(\boldsymbol{\tau}, v)=-\sum_{K \in \mathcal{P}} \int_{\partial K} v \boldsymbol{\tau} \cdot \mathbf{n} d \boldsymbol{x}, \quad X=\tilde{X}=\mathbf{W}^{0}, \quad X_{\mathrm{rm}}=\{0\} \\
M=H^{1 / 2}(\mathcal{E}), \quad l(\boldsymbol{\tau})=-\int_{\Omega} \mathcal{C}^{-1} \boldsymbol{\sigma}^{f} \cdot \boldsymbol{\tau} d \boldsymbol{x}, \quad \sigma(v)=\sum_{K \in \mathcal{P}} \int_{\partial K} v \boldsymbol{\sigma}^{f} \cdot \mathbf{n} d \boldsymbol{x} .
\end{gathered}
$$

The operators $T: H^{1 / 2}(\mathcal{E}) \rightarrow \mathbf{W}^{0}$ and $\hat{T}:\left(\mathbf{W}^{0}\right)^{\prime} \rightarrow \mathbf{W}^{0}$ are such that, given $v \in H^{1 / 2}(\mathcal{E})$, (18)

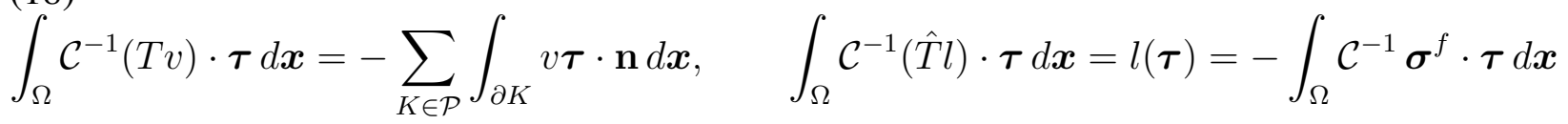
for all $\tau \in \mathbf{W}^{0}$. The MHM method seeks $u \in H^{1 / 2}(\mathcal{E})$ such that

$$
\sum_{K \in \mathcal{P}} \int_{\partial K} v(T u) \cdot \mathbf{n} d \boldsymbol{x}=\sum_{K \in \mathcal{P}} \int_{\partial K} v \boldsymbol{\sigma}^{f} \cdot \mathbf{n} d \boldsymbol{x}+\sum_{K \in \mathcal{P}} \int_{\partial K} v \hat{T} l \cdot \mathbf{n} d \boldsymbol{x} \quad \text { for all } v \in H^{1 / 2}(\mathcal{E}) .
$$

4.4.2. Discretization. In the spirit of Remark 5, we consider a discretization $M_{h} \subset M$, using here a notation more adapted to the present problem. Let $\left\{\psi_{i}\right\}_{i=1}^{\operatorname{dim} M_{h}}$ be a basis for $M_{h}$, and $\boldsymbol{\sigma}_{i}=-T \psi_{i}$. If $u^{h}=\sum_{i=1}^{\operatorname{dim} M_{h}} u_{i} \psi_{i}$, then, from (5), $\boldsymbol{\sigma}=\sum_{i=1}^{\operatorname{dim} M_{h}} u_{i} \boldsymbol{\sigma}_{i}+\hat{T} l$. Note that $\boldsymbol{\sigma}_{i}$ solve

$$
\int_{K} \mathcal{C}^{-1} \boldsymbol{\sigma}_{i} \cdot \boldsymbol{\tau} d \boldsymbol{x}=\int_{\partial K} \psi_{i} \boldsymbol{\tau} \cdot \mathbf{n} d \boldsymbol{x}
$$

for all $\boldsymbol{\tau} \in \mathbf{W}^{0}$ and $K \in \mathcal{P}$. Thus $\boldsymbol{\sigma}_{i}=\mathcal{C} \nabla \phi_{i}$, where $\phi_{i}$ solves

$$
-\operatorname{div} \mathcal{C} \nabla \phi_{i}=0 \text { in } K, \quad \phi_{i}=\psi_{i} \text { on } \partial K .
$$

We now extend the basis functions $\psi_{i}$, defined on the mesh skeleton, to the whole domain $\Omega$. Let $\Psi_{i} \in \stackrel{\circ}{H}^{1}(\Omega)$ be such that $\left.\Psi_{i}\right|_{\mathcal{E}}=\psi_{i}$. It is clear that the extension is not unique, and we will explore such flexibility further on. Note that

$$
\begin{gathered}
\sum_{K \in \mathcal{P}} \int_{\partial K} \psi_{j}(T u) \cdot \mathbf{n} d \boldsymbol{x}=-\sum_{i=1}^{\operatorname{dim} M_{h}} u_{i} \int_{\Omega} \boldsymbol{\nabla} \Psi_{j} \cdot \boldsymbol{\sigma}_{i} d \boldsymbol{x}=-\sum_{i=1}^{\operatorname{dim} M_{h}} u_{i} \int_{\Omega} \boldsymbol{\nabla} \Psi_{j} \cdot \mathcal{C} \boldsymbol{\nabla} \phi_{i} d \boldsymbol{x}, \\
\sum_{K \in \mathcal{P}} \int_{\partial K} \psi_{j}\left(\boldsymbol{\sigma}^{f}+\hat{T} l\right) \cdot \mathbf{n} d \boldsymbol{x}=\int_{\Omega} \boldsymbol{\nabla} \Psi_{j} \cdot\left(\boldsymbol{\sigma}^{f}+\hat{T} l\right) d \boldsymbol{x}-\int_{\Omega} f \Psi_{j} d \boldsymbol{x} .
\end{gathered}
$$

Thus, from (19),

$\sum_{i=1}^{\operatorname{dim} M_{h}} u_{i} \int_{\Omega} \nabla \Psi_{j} \cdot \mathcal{C} \nabla \phi_{i} d \boldsymbol{x}=-\int_{\Omega} \boldsymbol{\nabla} \Psi_{j} \cdot\left(\boldsymbol{\sigma}^{f}+\hat{T} l\right) d \boldsymbol{x}+\int_{\Omega} f \Psi_{j} d \boldsymbol{x} \quad$ for $j=1, \ldots, \operatorname{dim} M_{h}$. 
Note that (21) corresponds to the discretization of (19), which is defined on the mesh skeleton. By the same token, (21) depends only on $\left\{\psi_{j}\right\}_{j=1}^{\operatorname{dim} M_{h}}$ (recall that $\psi_{j}$ is the trace of $\Psi_{j}$ ). Indeed, it suffices to fix each basis function $\psi_{j}$ at all element edges to uniquely define the unknowns $\left\{u_{i}\right\}_{i=1}^{\operatorname{dim} M_{h}}$. Given the freedom to define the basis functions at the elements interior, it seems that the elegant choice is to let $\psi_{i}=\phi_{i}$, see (20). With that and the aid of (18), the formulation (21) becomes

$$
\sum_{i=1}^{\operatorname{dim} M_{h}} u_{i} \int_{\Omega} \boldsymbol{\nabla} \phi_{j} \cdot \mathcal{C} \nabla \phi_{i} d \boldsymbol{x}=\int_{\Omega} f \phi_{j} d \boldsymbol{x} \quad \text { for } j=1, \ldots, \operatorname{dim} M_{h} .
$$

It turns out that (22) is actually the Multiscale Finite Element Method (MsFEM) $[9,14,25]$. Such characterization of the MsFEM eliminates the need to choose the basis functions in an $a d$ hoc manner, only the definition of $\psi_{j}$ is needed. Now the fact that the basis functions solve the operator is a consequence of the method, not its first step. This resonates with the way the Residual Free Bubble Method is developed, and the remark below explore such connection.

Remark 7. Note that (21) can be further simplified by choosing $\boldsymbol{\sigma}^{f}=\mathcal{C} \boldsymbol{\nabla} \theta$, where $\left.\theta\right|_{K} \in \stackrel{\circ}{H}^{1}(K)$ solve $-\operatorname{div} \mathcal{C} \nabla \theta=f$ inside all elements $K \in \mathcal{P}$. Indeed, in such case, $\hat{T} l=0$. This is exactly the system solved by the "non-bubble" part of the Residual Free Bubble (RFB) method [8, 18, 31]. Thus, over the edges, both methods coincide — see [7,31] for further details.

\section{A “New” Discontinuous Enrichment-Multiscale Hybrid Mixed Method}

Often, for problems posed in heterogeneous media, the "macroscopic information" is of most interest, and such information can be well-approximated by polynomial functions. The trouble is, traditional methods with polynomial basis functions do not perform well for such problems. In general, the MHM method does not yield such approximation at once either, since $X_{\text {rm }}$ might be too simple or even the zero space, and only after the primal variable postprocessing a good approximation comes about.

The Residual Free Bubble (RFB) method [8, 18,31] tries to overcome such shortcoming by adding bubbles to the finite element spaces, and shares similarities to the MsFEM, presented in a dual hybrid form in Subsection 4.4. Using a hybrid formulation that is closer to the original proposition of the MHM method, the Discontinuous Enrichment Method (DEM) [17], also enriches polynomial spaces with functions that are local solutions to the PDE under consideration. This time however, the added space is nonconforming. 
5.1. The method. We now combine the ideas behind the DEM and MHM method, in a method that we call DE-MHM, aiming to preserve the good properties of both approaches. Starting with (1), we decompose $X$ in the three-field $X=X_{\mathrm{rm}} \oplus X_{\mathrm{p}} \oplus X_{\mathrm{e}}$, where the definition of $X_{\mathrm{rm}}$ is as in (2). We assume that $X_{\mathrm{p}} \subset \operatorname{Kern} B$, and $X_{\mathrm{e}}$ is, for instance, the orthogonal complement of $X_{\mathrm{rm}} \oplus X_{\mathrm{p}}$ with respect to the $X$-inner-product. In practice, $X_{\mathrm{p}}$ would be a space of continuous piecewise polynomials, and $X_{\mathrm{e}}$ a space of discontinuous functions that undergo static condensation.

We search then for $\left(u_{\mathrm{rm}}+u_{p}+u_{e}, \eta\right) \in X \times M$, where $u_{\mathrm{rm}} \in X_{\mathrm{rm}}, u_{p} \in X_{\mathrm{p}}$, and $u_{e} \in X_{\mathrm{e}}$ are such that

$$
\begin{aligned}
& b\left(v_{\mathrm{rm}}, \eta\right) \quad=l\left(v_{\mathrm{rm}}\right) \quad \text { for all } v_{\mathrm{rm}} \in X_{\mathrm{rm}}, \\
& a\left(u_{p}, v_{p}\right)+a\left(u_{e}, v_{p}\right) \quad=l\left(v_{p}\right) \quad \text { for all } v_{p} \in X_{\mathrm{p}} \text {, } \\
& a\left(u_{p}, v_{e}\right)+a\left(u_{e}, v_{e}\right)+b\left(v_{e}, \eta\right)=l\left(v_{e}\right) \quad \text { for all } v_{e} \in X_{\mathrm{e}} \\
& b\left(u_{\mathrm{rm}}, \mu\right)+b\left(u_{e}, \mu\right) \quad=\sigma(\mu) \quad \text { for all } \mu \in M \text {. }
\end{aligned}
$$

Similarly to (4), we define $T_{e}: M \rightarrow X_{\mathrm{e}}, T_{e}^{p}: X_{\mathrm{p}} \rightarrow X_{\mathrm{e}}$ and $\hat{T}_{e}: X \rightarrow X_{\mathrm{e}}$ such that

$$
a\left(T_{e} \eta, v_{e}\right)=b\left(v_{e}, \eta\right), \quad a\left(T_{e}^{p} u_{p}, v_{e}\right)=a\left(u_{p}, v_{e}\right), \quad a\left(\hat{T}_{e} l, v_{e}\right)=l\left(v_{e}\right), \quad \text { for all } v_{e} \in X_{\mathrm{e}}
$$

Then, from the third equation of (23):

$$
u_{e}=-T_{e} \eta-T_{e}^{p} u_{p}+\hat{T} l .
$$

Replacing in (23), we have from the first and last equations that

$$
\begin{aligned}
b\left(v_{\mathrm{rm}}, \eta\right)+b\left(u_{\mathrm{rm}}, \mu\right)+b\left(-T_{e} \eta, \mu\right)=b\left(T_{e}^{p} u_{p}, \mu\right)+l\left(v_{\mathrm{rm}}\right)+ & \sigma(\mu)-b(\hat{T} l, \mu) \\
& \quad \text { for all }\left(v_{\mathrm{rm}}, \mu\right) \in X_{\mathrm{rm}} \times M .
\end{aligned}
$$

Note that (26) is of the form (6), but since its right hand side depends on $u_{p}$, we perform yet another round of static condensation and write

$$
\left(u_{\mathrm{rm}}, \eta\right)=\left(S_{X} u_{p}+\hat{S}_{X}(\sigma, l), S_{M} u_{p}+\hat{S}_{M}(\sigma, l)\right),
$$

where $\left(S_{X} u_{p}, S_{M} u_{p}\right)$ and $\left(\hat{S}_{X}(\sigma, l), \hat{S}_{M}(\sigma, l)\right)$ are the solutions of the mixed problem (26) with $b\left(T_{e}^{p} u_{p}, \mu\right)$ and $l\left(v_{\mathrm{rm}}\right)+\sigma(\mu)-b(\hat{T} l, \mu)$ as the right hand side. 
Finally, using the second equation in (23), we gather the final equation

$$
\begin{array}{r}
a\left(u_{p}, v_{p}\right)-a\left(T_{e} S_{M} u_{p}, v_{p}\right)-a\left(T_{e}^{p} u_{p}, v_{p}\right)=l\left(v_{p}\right)-a\left(\hat{T} l, v_{p}\right)+a\left(T_{e} \hat{S}_{M}(\sigma, l), v_{p}\right) \\
\text { for all } v_{p} \in X_{\mathrm{p}} .
\end{array}
$$

The principal part of the solution is then $u_{\mathrm{rm}}+u_{p}$. Of course, $u_{e}$ can also be added if necessary.

An example of particular interest is for the Darcy problem (16) with an oscillatory tensor and based on the primal hybrid formulation, with $X_{\mathrm{p}}$ as the space of continuous piecewise polynomials.

Several remarks are now in order. First, the well-posedness of (28) and (1) are equivalent. In terms of implementation, as long as $X_{\mathrm{p}}$ is finite dimensional, the only discretization needed to solve (28), is that of the operators $T_{e}, T_{e}^{p}, S_{M}, \hat{T}$ and $\hat{S}_{M}$. Otherwise, a subspace of $X_{\mathrm{p}}$ is necessary. Regarding computational aspects, solving (26) is equivalent to solve the MHM method final system (6), and the local problems (24) are equivalent to (4). And size of the extra system (28), not present in the MHM method, depends only on the dimension of $X_{\mathrm{p}}$. A final remark concerns the possibility of considerable savings in terms of computer memory, since it is no longer necessary to store the solutions of problems (24) for postprocessing, as in the MHM method. Indeed, after these solutions are obtained, they can be used to assemble the matrices in (28) and discarded.

\section{REFERENCES}

[1] Rodolfo Araya, Christopher Harder, Diego Paredes, and Frédéric Valentin, Multiscale hybrid-mixed method, SIAM J. Numer. Anal. 51 (2013), no. 6, 3505-3531.

[2] Todd Arbogast, Gergina Pencheva, Mary F. Wheeler, and Ivan Yotov, A multiscale mortar mixed finite element method, Multiscale Model. Simul. 6 (2007), no. 1, 319-346.

[3] Todd Arbogast and Hailong Xiao, A multiscale mortar mixed space based on homogenization for heterogeneous elliptic problems, SIAM J. Numer. Anal. 51 (2013), no. 1, 377-399.

[4] D. N. Arnold and F. Brezzi, Mixed and nonconforming finite element methods: implementation, postprocessing and error estimates, RAIRO Modél. Math. Anal. Numér. 19 (1985), no. 1, 7-32 (English, with French summary).

[5] Daniele Boffi, Franco Brezzi, and Michel Fortin, Mixed finite element methods and applications, Springer Series in Computational Mathematics, vol. 44, Springer, Heidelberg, 2013.

[6] Franco Brezzi and Michel Fortin, Mixed and hybrid finite element methods, Springer Series in Computational Mathematics, vol. 15, Springer-Verlag, New York, 1991.

[7] F. Brezzi and L. D. Marini, Augmented spaces, two-level methods, and stabilizing subgrids, Internat. J. Numer. Methods Fluids 40 (2002), no. 1-2, 31-46. ICFD Conference on Numerical Methods for Fluid Dynamics (Oxford, 2001). 
[8] F. Brezzi, L. P. Franca, and A. Russo, Further considerations on residual-free bubbles for advective-diffusive equations, Comput. Methods Appl. Mech. Engrg. 166 (1998), no. 1-2, 25-33.

[9] C.-C. Chu, I. G. Graham, and T.-Y. Hou, A new multiscale finite element method for high-contrast elliptic interface problems, Math. Comp. 79 (2010), no. 272, 1915-1955.

[10] Philippe G. Ciarlet, The finite element method for elliptic problems, North-Holland Publishing Co., AmsterdamNew York-Oxford, 1978. Studies in Mathematics and its Applications, Vol. 4.

[11] Bernardo Cockburn and Jayadeep Gopalakrishnan, Error analysis of variable degree mixed methods for elliptic problems via hybridization, Math. Comp. 74 (2005), no. 252, 1653-1677 (electronic).

[12] Bernardo Cockburn, Jayadeep Gopalakrishnan, and Raytcho Lazarov, Unified hybridization of discontinuous Galerkin, mixed, and continuous Galerkin methods for second order elliptic problems, SIAM J. Numer. Anal. 47 (2009), no. 2, 1319-1365.

[13] Ramon Codina and Santiago Badia, On the design of discontinuous Galerkin methods for elliptic problems based on hybrid formulations, Comput. Methods Appl. Mech. Engrg. 263 (2013), 158-168.

[14] Yalchin Efendiev and Thomas Y. Hou, Multiscale finite element methods, Surveys and Tutorials in the Applied Mathematical Sciences, vol. 4, Springer, New York, 2009. Theory and applications.

[15] Yalchin Efendiev, Raytcho Lazarov, and Ke Shi, A multiscale HDG method for second order elliptic equations. Part I. Polynomial and homogenization-based multiscale spaces, arXiv (2013), available at 1310.2827.

[16] Alexandre Ern and Jean-Luc Gurmond, Theory and practice of finite elements, Applied Mathematical Sciences, vol. 159, Springer-Verlag, New York, 2004.

[17] Charbel Farhat, Isaac Harari, and Leopoldo P. Franca, The discontinuous enrichment method, Comput. Methods Appl. Mech. Engrg. 190 (2001), no. 48, 6455-6479.

[18] L. P. Franca and A. Russo, Deriving upwinding, mass lumping and selective reduced integration by residual-free bubbles, Appl. Math. Lett. 9 (1996), no. 5, 83-88.

[19] A. Francisco, V. Ginting, F. Pereira, and J. Rigelo, Design and Implementation of a Multiscale Mixed Method Based on a Nonoverlapping Domain Decomposition Procedure, Mathematics and Computers in Simulation (2013).

[20] Roland Glowinski and Mary Fanett Wheeler, Domain decomposition and mixed finite element methods for elliptic problems, Partial Differential Equations (Paris, 1987), SIAM, Philadelphia, PA, 1988, pp. 144-172.

[21] Peter Hansbo, Carlo Lovadina, Ilaria Perugia, and Giancarlo Sangalli, A Lagrange multiplier method for the finite element solution of elliptic interface problems using non-matching meshes, Numer. Math. 100 (2005), no. 1, 91115.

[22] Christopher Harder, Alexandre Madureira, and Frédéric Valentin, A Hybrid-Mixed Method for Elasticity in Two and Three-Dimensions (2013), submitted.

[23] Christopher Harder, Diego Paredes, and Frédéric Valentin, A family of multiscale hybrid-mixed finite element methods for the Darcy equation with rough coficients, J. Comput. Phys. 245 (2013), 107-130.

[24]___ On a Multiscale Hybrid-Mixed Method for Advective-Reactive Dominated Problems with Heterogenous Coefficients (2013), submitted. 
[25] Thomas Y. Hou, Numerical approximations to multiscale solutions in partial differential equations, Frontiers in numerical analysis (Durham, 2002), Universitext, Springer, Berlin, 2003, pp. 241-301.

[26] G. C. Hsiao, E. Schnack, and W. L. Wendland, Hybrid coupled finite-boundary element methods for elliptic systems of second order, Comput. Methods Appl. Mech. Engrg. 190 (2000), no. 5-7, 431-485.

[27] Qi-ya Hu, Guo-ping Liang, and Jin-zhao Liu, Construction of a preconditioner for domain decomposition methods with polynomial Lagrangian multipliers, J. Comput. Math. 19 (2001), no. 2, 213-224.

[28] Alexandre Madureira and Frédéric Valentin, A Locking free primal hybrid method for the Reissner-Mindlin plate model (2013), in preparation.

[29] Alfio Quarteroni and Alberto Valli, Numerical approximation of partial differential equations, Springer Series in Computational Mathematics, vol. 23, Springer-Verlag, Berlin, 1994. MR1299729 (95i:65005)

[30] J. E. Roberts and J.-M. Thomas, Mixed and hybrid methods, Handbook of numerical analysis, Vol. II, Handb. Numer. Anal., II, North-Holland, Amsterdam, 1991, pp. 523-639.

[31] Giancarlo Sangalli, Capturing small scales in elliptic problems using a residual-free bubbles finite element method, Multiscale Model. Simul. 1 (2003), no. 3, 485-503 (electronic).

[32] Wikipedia, Embarrassingly parallel (2013).

Departamento de Matemática Aplicada e Computacional, laboratório Nacional de ComputaÇÃo Científica, Av. Getúlio Vargas, 333, 25651-070 Petrópolis - RJ, Brazil

E-mail address: almelncc.br

Fundação getúlio Vargas, Praia de Botafogo 190, Rio de Janeiro - RJ, Brazil

E-mail address: alexandre.madureiraefgv.br 